\title{
Prophylactic Effect of Simultaneous Placement of Mesh on Incidence of Parastomal Hernia After Mile's Surgical Resection of Colorectal Cancer: A Prospective Study
}

\section{Xu Gao}

Department of general surgery, Beijing Luhe Hospital, Capital Medical University, 82 Xinhua South Road, Tongzhou District, Beijing, China

\section{Ruo-Fan Li}

Department of general surgery, Beijing Luhe Hospital, Capital Medical University, 82 Xinhua South Road, Tongzhou District, Beijing, China

\section{Li-Xin Sun ( $\square$ lixinsun126@gmail.com )}

Department of general surgery, Beijing Luhe Hospital, Capital Medical University, 82 Xinhua South Road, Tongzhou District, Beijing, China

\section{Zuo-Jun Liu}

Department of general surgery, Beijing Luhe Hospital, Capital Medical University, 82 Xinhua South Road, Tongzhou District, Beijing, China

\section{Guang-Jian Tian}

Department of general surgery, Beijing Luhe Hospital, Capital Medical University, 82 Xinhua South Road, Tongzhou District, Beijing, China

\section{Hui Qi}

Department of general surgery, Beijing Luhe Hospital, Capital Medical University, 82 Xinhua South Road, Tongzhou District, Beijing, China

\section{Xiao-Bin Li}

Department of general surgery, Beijing Luhe Hospital, Capital Medical University, 82 Xinhua South Road, Tongzhou District, Beijing, China

\section{Research Article}

Keywords: colorectal cancer, hernia, hernia mesh, incidence, parastomal hernia, surgery

Posted Date: February 26th, 2021

DOl: https://doi.org/10.21203/rs.3.rs-243315/v1 
License: (c) (i) This work is licensed under a Creative Commons Attribution 4.0 International License. Read Full License 


\section{Abstract}

Background: To assess the prophylactic effect of simultaneous placement of mesh and incidence of parastomal hernia (PSH) after abdominoperineal resection of rectal cancer.

Methods: This study included real world data of 56 surgically resected colorectal cancer (CRC) patients who were consecutively assigned to 2 groups: control (no mesh, $n=32$ ) and experimental (received mesh, $\mathrm{n}=24$ ). An artificial patch was placed under the tunica vaginalis of rectus abdominis for patients in experimental group whereas the control group received routine sigmoidostomy. The median follow-up time was $>20$ months. Difference in hazards function was analyzed by cox regression analysis and results were expressed as hazard ratio (HR) and 95\% confidence interval (Cl). A P-value $<0.05$ was considered as significant.

Results: Post-operative incidence rate of PSH was lower in experimental (41.7 \%) than in control group (71.9 \%; $\mathrm{P}=0.045)$. PSH postoperative time in experimental group was significantly delayed than control group (48 months vs 10 months; $\mathrm{P}<0.001$ ). The risk of progression from $\mathrm{H} 1$ to $\mathrm{H} 2$ was less in experimental group than control group ( $49.28 \%$ vs. $60.86 \%$; $P=0.14)$.

Conclusions: Prophylactic mesh placement significantly prolonged post-operative time to recurrence and incidence rate of PSH. The incidence of recurrence of $\mathrm{H} 2$ (severe PSH) requiring secondary surgical repair was reduced.

\section{Background}

Colorectal cancer (CRC) is the third most common malignancy worldwide with its incidence increasing every year., However, its mortality is comparatively lower than that of other cancers (1). Even with advancements in surgical techniques and instruments, patients with rectal cancer still suffer permanent colon stoma. With the progress of radical tumor surgery and postoperative drug therapy, the 5-year survival rate of colorectal cancer is gradually increasing along with the chances of occurrence of new complications. Parastomal hernia (PSH) is a common complication of end-colostomy (2) which affects the patient's quality of life after surgery, and even lead to a particularly life-threatening hernia. As per reports from Israelsson et.al (3), the 5-year incidence of PSH was $30 \%-50 \%$, whereas it was $15 \%$ as reported elsewhere (4). Lopez-Cano et al. also reported higher incidence of PSH (93.8\%) (5). In China, with the increasing time in future, the rate of PSH can reach to $100 \%$ (6). A study reported that at 5-years follow-up the rate of PSH after Miles surgery reached to $72.88 \%$ (7) .

Apart from significantly impact patients' quality of life, $\mathrm{PSH}$ also cause a variety of complications such as pain, bleeding, bowel obstruction, and bowel strangulation (8).

In this view, a number of methods have been developed that can be employed in corrective surgery. Surgical techniques for repairing PSH include local fixation, resiting the stoma, and prosthetic mesh repair (9). However, the recurrence rate remains high after surgical treatment of PSH (10). Nevertheless, 
the only technique that has been examined in detail in prospective randomized controlled trials is the use of prophylactic mesh at the time of stoma formation. A meta-analysis (11) on prophylactic application of mesh showed that the incidence of PSH was $12.5 \%$ in treatment group compared to no mesh group (53\%), but there was no difference in postoperative complications. In China, most of the reports are from those with re-operation after herniotomy. Yu Yifeng (12) reported that there was no occurrence of stoma hernia after one and a half years when prophylactic mesh was used in intraperitoneal operation. Having said so, this method of placing Special anti-adhesion patches inside the abdominal cavity might not be cost effective (13), and requires latest technology and equipment, limiting its routine use in clinical set up. Besides, when PSH occurs after surgery, (14), the literature reports that $30 \%$ of patients require postoperative intervention. and most of these are severe $\mathrm{PSH}$, which can easily lead to a hernia that is life-threatening. .How to identify this part of patients as early as possible also require special attention in clinical work (14).

At present, application of this new technique is less across hospitals in China. Additionally, lack of set unified performing standards and small sample sizes in studies reported, it is difficult to evaluate the effectiveness of postoperative effect of prophylactic mesh. In view of this uncertainty, we designed prospective study to evaluate the effects of using a prophylactic mesh around the stoma. The primary

aim was to evaluate the PSH rate in both groups. Our hypothesis was that prophylactic mesh reduced the incidence of PSH after abdominoperineal resection of rectal cancer. A PSH classification was designed to determine the necessity of secondary hernia repair in selected high-risk PSH patients.

\section{Methods}

This was a non-randomized, interventional study where data was collected prospectively of 77 surgically resected CRC patients from January 2014 to December 2018 at Department of general surgery, Beijing Luhe Hospital, Capital Medical University, Beijing, China. They were consecutively assigned to two groups: control (no preset mesh, $n=32$ ) and experimental (received preset mesh, $n=24$ ) (Figure 1). The choice of placement of mesh or undergoing routine sigmoidostomy was determined by the clinical experience of physicians and by the patient preference.

Patients with colorectal cancer who needed to undergo Mile's operation, patients without severe heart failure, lung and kidney failure, with follow-up time more than 6 months and those willing to provide written informed consent were included in this study. On the other hand, patients with any major organ dysfunction/disorder and thus, cannot tolerate surgery were excluded. The primary endpoints were to evaluate incidence rate and time to recurrence of $\mathrm{PSH}$ were observed.

The study protocol was approved by Ethics Committee (Institutional Review Board) of Beijing Luhe Hospital, Capital Medical University, China (Study number: 2020-LHKY-033-01) and conducted in accordance with the Declaration of Helsinki. All patients provided written informed consent before participation in the study.

\section{Disease diagnosis and PSH classification}


Parastomal hernia is a type of abdominal incisional hernia occurring adjacent to or located near the stoma. It is an abnormal protrusion of abdominal contents caused by the defect of abdominal wall caused by enterostomy (14). The classification of PSH is defined as follows: H0, without parastomal hernia; $\mathrm{H} 1$, sliding hernia where the hernia goes into intestine next to the fistula and can return to the abdominal cavity by itself, does not require surgical intervention; $\mathrm{H} 2$, severe complicated hernia where the hernia goes into the intestine beside the fistula however cannot return to the abdominal cavity by itself, there is a risk of intestinal necrosis caused by obstruction and require second operation (Figure 2).

\section{Surgical Regimen}

Patients in the interventional group underwent rectal cancer resection using laparoscopy according to the principle of TME. A single fiber polypropylene non absorbable mesh, produced by Italian company HERNIAMESH (Model: H52535) was used. The rectum, sigmoid colon and surrounding lymph tissue were separated and resected. The left colon artery was reserved to clean the lymph tissue adjacent to the inferior mesenteric artery (253 group lymph nodes). $5 \%$ lodophor was used to disinfect the cutting edge after the tumor was cut off by the cutter. The tumor and distal rectum were pulled out from the anus. The skin was incised obliquely at the left lower abdominal cavity (The location of the stoma was selected by the stoma therapist, and all of them were made by lateral rectus abdominis) The length of the stoma was about $3 \mathrm{~cm}$. The subcutaneous and external oblique aponeurosis were separated layer by layer. The oblique muscle and transverse muscle of abdomen were separated bluntly, and the round preperitoneal space was about 13-15 cm in diameter. One circular mesh with an outer diameter of $13 \mathrm{~cm}$ and an inner diameter of $4 \mathrm{~cm}$ was laid in the preperitoneal space. The central side of the mesh was intermittently sutured with peritoneum and abdominal muscle layer with $2 \times 0$ absorbable suture. The peritoneum was incised and the peritoneum was sutured with the muscular layer of abdominal wall. The mesh was wrapped in it to make the central edge of the mesh peritoneum, Peritonealization of the central lateral margin of the patch was done so as to avoid the stricture of incision/scar caused by the direct contact between the mesh and intestinal tube. The proximal end of sigmoid colon was raised from the incision through the center of the mesh. The intestinal wall of colostomy was intermittently sutured with peritoneum, aponeurosis of external oblique muscle and skin of abdominal wall. The closed end of colon was incised with electric knife to check that the stoma is unobstructed, the blood supply is good, and the external ostomy bag is connected.

On the other hand, patients in the control group underwent laparoscopic assisted radical resection of rectal cancer, routine fistulas of the sigmoid colon (lateral rectus abdominis and a fistula of about $3 \mathrm{~cm}$ ), without separating the lacunae between the submuscular layer of the abdominal wall and the anterior peritoneum.

\section{Post-operative Observation}

ALL of these patients were taken care by full-time trained and certified stoma nurse who observed them for any signs of defecation due to stoma, to avoid skin infection around the stoma, and to help expand 
anus regularly. After discharge, the patients were followed up by specialists in outpatient department to observe whether there were complications such as fistula infection, retraction, stenosis and prolapse.

\section{Statistical analysis}

Continuous variables were compared using independent sample t-test and the normally distributed data were expressed as mean \pm standard deviation (SD). Categorical data were expressed as $\mathrm{n}$ (proportion). Comparisons between categorical variables were performed using Chi-square test. The incidence of hernia between experimental and control group at the end of the study period was calculated. Chi-square test and $\mathrm{Z}$ statistics was calculated to check whether the incidence for recurrence of hernia $(\mathrm{H} 1, \mathrm{H} 2)$ between the two groups were significant, while risk of progression from $\mathrm{H} 1$ to $\mathrm{H} 2$ was calculated using Fischer-exact test. KM estimator function and log rank test was used to compare the differences in survival curves among the two groups. Further, difference in hazards function was analyzed by cox regression analysis and results were expressed as hazard ratio (HR) and 95\% confidence interval (Cl). A P-value $<0.05$ was considered as significant. All analyses were performed using $\mathrm{R}$ software 3.6.2.

\section{Results}

In total, 77 patients (53 controls, 24 experimental) were initially enrolled into this study. After excluding patients with follow-up less than 6 months, data for analysis consisted of 32 patients ( 12 female, 20 male) in control and 24 (6 female, 18 male) in experimental group (Figure 1). The mean age of patients in both the groups was comparable (mean \pm SD: control: $65.42 \pm 12.9$ years and experimental $65.53 \pm 11.8$ years. The AJCC staging for TNM classification showed no significant difference between the two treatment groups $(P=0.7304)$ (Table 1$)$.

\section{Incidence rate of PSH after operation}

The recurrence of PSH $(\mathrm{H} 1, \mathrm{H} 2)$ was lower in experimental group than in control group $(41.7 \% \mathrm{vs.} 71.9 \%)$ with statistically significant differences between the two groups $(P=0.045)$. The incidence of $\mathrm{H} 1$ hernia after operation was similar in experimental group than in control group (29.17\% vs. $28.13 \%)$, though there was no statistical difference $(\mathrm{P}=0.999)$. However, the proportion of patients with occurrence of $\mathrm{H} 2$ hernia (severe) after operation was lower in patients belonging to experimental group than in control group with statistically significant results $(12.50 \%$ vs. $43.75 \%$; $P=0.026)$.

\section{Time of recurrence of postoperative PSH}

Prophylactic use of mesh prolonged the median time for postoperative PSH in experimental group (48 months) and was statistically significant between the two groups (control: 10 months, $P=0.00031$, Figure 3). It was also seen that patients in experimental group were at significantly lower risk of PSH than those without the mesh placement (HR: 0.24, 95\% Cl: 0.1, 0.55; $\mathrm{P}<0.001)$.

\section{Cumulative incidence of PSH}


It was seen that control group had numerically higher incidence of PSH as compared to experimental group. It was also seen that time for incidence of hernia was longer for experimental group than for the control group (Figure 4).

\section{Survival time}

Most of the patients were censored before the follow-up period and hence median survival time was not reached in both the treatment groups $(P=0.34)$ (Figure 5$)$. However, though not statistically significant, the risk of death in the experimental group was higher (HR: 2.7, 95\% Cl: $0.31,24 ; \mathrm{P}=0.36$ ) than in the control group owing to the longer average follow-up in the experimental group (32.5 months) compared to the control group (20.19 months

\section{Incidence of progression from $\mathrm{H} 1$ to $\mathrm{H} 2$ stage of PSH}

The incidence of progression from $\mathrm{H} 1$ to $\mathrm{H} 2$ stage was statistically significant with lesser risk to progression in experimental group patients than in control group $(49.28 \% \mathrm{vs.} 60.86 \% ; \mathrm{P}=0.14)$.

\section{Safety}

None of the patients in either the control or experimental groups experienced any post-operative complications such as pain, bleeding, intestinal obstruction or intestinal necrosis.

\section{Discussion}

In this study, the prophylactic effect of simultaneous placement of mesh beside the stoma during CRC radical resection to prevent $\mathrm{PSH}$ seemed beneficial rather than routine sigmoidostomy in prolonging time to recurrence for PSH. Our findings are in line with previous studies wherein the incidence rate for recurrence for PSH in patients was significantly lower than in patients without the mesh. Going by the estimates, the recurrence rate has been shown to be almost nine times higher without mesh $(15,16)$. Pioneering reports on using mesh for repairs was published by Hopkins and Trento (16), however the technique was limited in its clinical use in light of intestinal erosions and infections $(16,17)$. However, effectiveness and safety of this procedure still remains controversial.

In a prospective study involving 344 patients undergoing PSH repair using mesh, the recurrence rate was a mere $2.1 \%$, and no complications were reported during the follow up, which could be attributed to the mesh (18). On contrary, results from a retrospective cohort involving ventral hernia repair showed frequent occurrence of intestinal obstruction secondary to adhesions with PVDF than when with Parietex mesh (19). Thus, it is of foremost importance and necessary to standardize the surgical method and approach while taking in long-term outcomes to understand the exact benefits of mesh use $(20,21)$.

The risk of PSH increases substantially when the stoma is created, because the abdominal wall, which was otherwise undamaged, now becomes defective (22). Advanced age, obesity, smoking, ostomy size, ostomy location, malnutrition, diabetes, lung disease, hormone drug use is all high-risk factors for 
postoperative PSH. For every increase in the patient age, the risk of developing PSH increases by $4 \%$ (23). Studies have shown that BMI $\left(\mathrm{kg} / \mathrm{m}^{2}\right)$ greater than 25 can increase the incidence of parastomal hernia, and gender is an independent risk factor for the occurrence and development of parastatal hernia $(24,25)$.

The level of stability provided by underlying structure of the muscles of the abdomen which, when applied in creating the stoma, can lower the potential for hernias to develop (22). Reduction in PSH risk can be achieved through careful and sufficient preparation prior to surgery, opting an optimal stoma location, and identifying patients who are at the greatest risk of PSH (22). During follow up, although these procedures have promised lower incidence of PSH when compared to a non-prophylactic method, but due to the limitations of methodology and absence of any standardization, it is necessary to remain cautious when interpreting any results thus far (26-29).

The prophylactic use of a mesh when the stoma is created is demonstrated in several studies; nonetheless this does not support the fact of using this approach to prevent $\mathrm{PSH}$. To date, various randomized controlled trials have assessed these comparisons (30-41). These studies utilized a wide variety of meshes and surgical methods, using open and laparoscopic procedures to place various mesh styles in different positions. Thus, because of a lack of consistency in the other variables involved, it can be difficult to draw conclusions when comparing particular factors.

In our study, the fact that the incidence rate of severe postoperative PSH was different between the two groups $(41.7 \%$ vs. $71.9 \%)$ show that placement of mesh can reduce the probability of second operation in the future, reduce trauma, cost, and surgical risk. While compared with previous reports of $10-20 \%$ hernia incidence, our overall incidence was high due to inclusion of $\mathrm{H} 1$ and $\mathrm{H} 2$ types). Nevertheless, in previous PSH studies, the main study was the $\mathrm{H} 2$ type with abdominal mass, which was basically the same as the incidence of type $\mathrm{H} 2(12.5 \%)$ in our experimental group (42). We analyzed that a considerable number of patients had $\mathrm{H} 1$ type hernia that progressed to $\mathrm{H} 2$ type hernia over time. This rate of progression was lower in the patients belonging to experimental group (49.28\%) compared to those in the control group was high (60.86\%). However, a considerable number of patients with $\mathrm{H} 1$ mild PSH hernia go undetected during the course of diagnosis and corresponding preventive measures are not taken to avoid further aggravation. These patients can be distinguished through our classification, and a series of measures can be taken in the future to slow their progress.

At the same time, our study has been to an extent successful in showcasing the benefits of placing a prophylactic mesh during the surgical resection in significantly prolonging the time for $\mathrm{PSH}$ recurrence (48 months vs. 10 months). It is noteworthy to mention that the onset time of 48 months in the current experimental group was higher than the median survival of 29.4 months after CRC surgery (43). This may also implicate that a considerable number of patients may not be affected by PSH in their future life. The 10 -year recurrence rate of incisional hernia is estimated to be $63 \%$ for conventional meshless suture repair and $32 \%$ for prosthetic mesh repairs (44). Because of the variability and poor-quality evidence in the previous studies, it is now suggested that although prophylactic mesh can be helpful in preventing $\mathrm{PSH}$, it is important to discuss the risks and benefits prior to surgery with patients. In future it will also be 
useful to establish techniques which can be performed easily by novice surgeons within a fair additional operative time and without unnecessary complexity (20).

A strength of our study is defining strict inclusion and exclusion criteria that allowed comparison between groups of patients undergoing surgery for the same indication by the same operating surgeons, using a uniform technique. In addition, the long follow-up time period (the longest follow-up time of the experimental group is 63 months with median follow-up time of 32.5 months; the longest follow-up time of the control group is 58 months with median follow-up time of 20.19 months), enabled us to represents the general state of most patients after this type of surgery, and thus can reflect the influence of the preset patch on the incidence of PSH. However, limitations are small sample size, loss to follow up and study design that is used to assess this approach. These might limit the extrapolation of these clinical findings.

In future, more studies with sufficient sample size and longer follow-up period along with clinical correlation would be useful in assessing the long-term effectiveness and safety of prophylactic mesh in preventing PSH development. Efforts must be made in using prophylactic mesh in patients at high risk of post-operative PSH in routine clinical practice.

\section{Conclusion}

Implantation of preset mesh in surgically resected patients with CRC reduced the incidence of parastomal hernia after operation, prolonged the survival time among patients with the probability of reduced recurrence of hernia in future. Incidence of severe PSH $(\mathrm{H} 2)$ requiring secondary surgical intervention was also significantly reduced. In the overall study, there were no complications such as stoma infection, stoma subcutaneous effusion, ostomy stenosis, intestinal obstruction, and intestinal leakage with the placement of extraperitoneal mesh with controllable risks. Also, when compared with the intra-abdominal patch, the overall cost with this technique was not significantly increased with a marginal increase in the overall operation time. Thus, prophylactic placement of mesh will be beneficial in patients to control the postoperative complications along with improving the quality of life of patients in surgical resection patients with CRC.

\section{Declarations}

\section{Ethics approval and consent to participate}

The study protocol was approved by Ethics Committee (Institutional Review Board) of Beijing Luhe Hospital, Capital Medical University, China (Study number: 2020-LHKY-033-01) and conducted in accordance with the Declaration of Helsinki. All patients provided written informed consent before participation in the study.

\section{Consent for publication}


Not applicable

\section{Availability of data and materials}

All data used in this study will be available from the corresponding author upon reasonable request.

\section{Competing interests}

The authors declare that they have no competing interests.

\section{Funding}

This work was supported by Luhe Hospital Medical Development Research Fund, Capital Medical University, China.

\section{Authors' contributions}

$X G$ and RL conceptualized, designed the work, acquired and interpreted the data, performed statistical analysis and drafted the manuscript. ZL and GT did pathologic examination and interpretation of pathologic data. XG, RL, LS, ZL, GT, HQ and XL critically revised the manuscript. LS provided technical and material support, and supervision. All authors read and approved the final manuscript.

\section{Acknowledgements}

The author would like to thank his wife Xiao-Juan Jing, his father Li-gang Gao, his mother Yan-e Li for their great support for execution of this work.

\section{References}

1. Ferlay J, Soerjomataram I, Dikshit R, Eser S, Mathers C, Rebelo M, et al. Cancer incidence and mortality worldwide: Sources, methods and major patterns in GLOBOCAN 2012: Globocan 2012. Int J Cancer. 2015 Mar 1;136(5):E359-86.

2. Hamada M, Tamura T, Sakamoto S. Laparoscopic treatment of parastomal hernia using sugerbaker technique with parietex composite mesh. Surg Endosc Other Interv Tech. 2013;27:S389.

3. Israelsson LA. Parastomal hernias. Surg Clin North Am. 2008 Feb;88(1):113-25, ix.

4. Janson AR, Jänes A, Israelsson LA. Laparoscopic stoma formation with a prophylactic prosthetic mesh. Hernia. 2010 Oct;14(5):495-8.

5. Lopez-Cano. Use ofa prosthetic mesh to prevent parastomal hernia during laparoscopic abdominoperineal resection囚a randomized controlled trial.

6. De-hong Yu. The Management of Intestinal Stomas. Beijing:People's Medical Publishing House. 2004;(179). 
7. Zhu Deyi, Wang Ting, Zuo Wei. Clinical analysis of related risk factors for paraostomy hernia formation after Miles rectal cancer[J] Journal of Wenzhou Medical University, 2015,45(5):372-375. Journal of Wenzhou Medical University. 2015;45(5):372-5.

8. Styliński R, Alzubedi A, Rudzki S. Parastomal hernia - current knowledge and treatment. wiitm. 2018;13(1):1-8.

9. Rieger N, Moore J, Hewett P, Lee S, Stephens J. Parastomal hernia repair. Colorect Dis. 2004 May;6(3):203-5.

10. Wara P. Parastomal hernia repair. An update. Minerva Chir. 2011 Apr;66(2):123-8.

11. Shabbir J, Chaudhary BN, Dawson R. A systematic review on the use of prophylactic mesh during primary stoma formation to prevent parastomal hernia formation. Colorectal Dis. 2012 Aug;14(8):931-6.

12. Yu Y, Feng Q, Li S. Experience of prevention and use of Bard\&apos;s parastomal hernia patch in abdominal perineum combined with radical resection of rectal cancer. Chinese Journal of clinicians (Electronic Edition). 2011;5(14):4287-8.

13. Slater NJ, Hansson BME, Buyne OR, Hendriks T, Bleichrodt RP. Repair of Parastomal Hernias with Biologic Grafts: A Systematic Review. J Gastrointest Surg. 2011 Jul;15(7):1252-8.

14. Martin L, Foster G. Parastomal hernia. Ann R Coll Surg Engl. 1996 Mar;78(2):81-4.

15. Gregg ZA, Dao HE, Schechter S, Shah N. Paracolostomy Hernia Repair: Who and When? Journal of the American College of Surgeons. 2014 Jun;218(6):1105-12.

16. Hopkins TB, Trento A. Parastomal Ileal Loop Hernia Repair with Marlex Mesh. Journal of Urology. 1982 Oct;128(4):811-2.

17. Hotouras A, Murphy J, Thaha M, Chan CL. The persistent challenge of parastomal herniation: a review of the literature and future developments. Colorectal Dis. 2013 May;15(5):e202-14.

18. Berger D, Bientzle M. Polyvinylidene fluoride: a suitable mesh material for laparoscopic incisional and parastomal hernia repair!: A prospective, observational study with 344 patients. Hernia. 2009 Apr;13(2):167-72.

19. Tandon A, Shahzad K, Pathak S, Oommen C, Nunes Q, Smart N. Parietex ${ }^{T M}$ Composite mesh versus DynaMesh ${ }^{\circledR}$-IPOM for laparoscopic incisional and ventral hernia repair: a retrospective cohort study. annals. 2016 Nov;98(8):568-73.

20. Antoniou SA, Agresta F, Garcia Alamino JM, Berger D, Berrevoet F, Brandsma H-T, et al. European Hernia Society guidelines on prevention and treatment of parastomal hernias. Hernia. 2018 Feb;22(1):183-98.

21. Brown C, Finch J. Which mesh for hernia repair? annals. 2010 May;92(4):272-8.

22. Techagumpuch A, Udomsawaengsup S. Update in parastomal hernia. Ann Laparosc Endosc Surg. 2019 Aug;4:75-75.

23. Pilgrim $\mathrm{CHC}$, Mclntyre R, Bailey M. Prospective audit of parastomal hernia: prevalence and associated comorbidities. Dis Colon Rectum. 2010 Jan;53(1):71-6. 
24. Donahue TF, Bochner BH, Sfakianos JP, Kent M, Bernstein M, Hilton WM, et al. Risk Factors for the Development of Parastomal Hernia after Radical Cystectomy. J Urol. 2014 Jun;191(6):1708-13.

25. Hong SY, Oh SY, Lee JH, Kim DY, Suh KW. Risk factors for parastomal hernia: based on radiological definition. J Korean Surg Soc. 2013 Jan;84(1):43-7.

26. Helgstrand F, Rosenberg J, Kehlet H, Jorgensen LN, Wara P, Bisgaard T. Risk of Morbidity, Mortality, and Recurrence After Parastomal Hernia Repair: A Nationwide Study. Diseases of the Colon \& Rectum. 2013 Nov;56(11):1265-72.

27. Williams NS, Hotouras A, Bhan C, Murphy J, Chan CL. A case-controlled pilot study assessing the safety and efficacy of the Stapled Mesh stomA Reinforcement Technique (SMART) in reducing the incidence of parastomal herniation. Hernia. 2015 Dec;19(6):949-54.

28. Canda AE, Terzi C, Agalar C, Egeli T, Arslan C, Altay C, et al. Preventing parastomal hernia with modified stapled mesh stoma reinforcement technique (SMART) in patients who underwent surgery for rectal cancer: a case-control study. Hernia. 2018 Apr;22(2):379-84.

29. Hotouras A, Murphy J, Power N, Williams NS, Chan CL. Radiological incidence of parastomal herniation in cancer patients with permanent colostomy: What is the ideal size of the surgical aperture? International Journal of Surgery. 2013 Jun;11(5):425-7.

30. Jänes A, Cengiz Y, Israelsson LA. Preventing Parastomal Hernia with a Prosthetic Mesh: A 5-Year Follow-up of a Randomized Study. World J Surg. 2009 Jan;33(1):118-21.

31. Serra-Aracil X, Bombardo-Junca J, Moreno-Matias J, Darnell A, Mora-Lopez L, Alcantara-Moral M, et al. Randomized, Controlled, Prospective Trial of the Use of a Mesh to Prevent Parastomal Hernia: Annals of Surgery. 2009 Apr;249(4):583-7.

32. Hammond TM, Huang A, Prosser K, Frye JN, Williams NS. Parastomal hernia prevention using a novel collagen implant: a randomised controlled phase 1 study. Hernia. 2008 Oct;12(5):475-81.

33. López-Cano M, Lozoya-Trujillo R, Quiroga S, Sánchez JL, Vallribera F, Martí M, et al. Use of a prosthetic mesh to prevent parastomal hernia during laparoscopic abdominoperineal resection: a randomized controlled trial. Hernia. 2012 Dec;16(6):661-7.

34. López-Cano M, Serra-Aracils X, Mora L, Sánchez-García JL, Jiménez-Gómez LM, Martí M, et al. Preventing Parastomal Hernia Using a Modified Sugarbaker Technique With Composite Mesh During Laparoscopic Abdominoperineal Resection: A Randomized Controlled Trial. Annals of Surgery. 2016 Dec;264(6):923-8.

35. Fleshman J, Branda ME, Sargent DJ, Boller AM, George VV, Abbas MA, et al. Disease-free Survival and Local Recurrence for Laparoscopic Resection Compared With Open Resection of Stage II to III Rectal Cancer: Follow-up Results of the ACOSOG Z6051 Randomized Controlled Trial. Annals of Surgery. 2019 Apr;269(4):589-95.

36. Târcoveanu E, Vasilescu A, Cotea E, Vlad N, Palaghia M, Dănilă N, et al. Parastomal hernias -- clinical study of therapeutic strategies. Chirurgia (Bucur). 2014 Apr;109(2):179-84.

37. Vierimaa M, Klintrup K, Biancari F, Victorzon M, Carpelan-Holmström M, Kössi J, et al. Prospective, Randomized Study on the Use of a Prosthetic Mesh for Prevention of Parastomal Hernia of 
Permanent Colostomy: Diseases of the Colon \& Rectum. 2015 Oct;58(10):943-9.

38. Lambrecht JR, Larsen SG, Reiertsen O, Vaktskjold A, Julsrud L, Flatmark K. Prophylactic mesh at endcolostomy construction reduces parastomal hernia rate: a randomized trial. Colorectal Dis. 2015 Oct;17(10):0191-7.

39. Brandsma H-T, Hansson BME, Aufenacker TJ, van Geldere D, Lammeren FMV, Mahabier C, et al. Prophylactic Mesh Placement During Formation of an End-colostomy Reduces the Rate of Parastomal Hernia: Short-term Results of the Dutch PREVENT-trial. Annals of Surgery. 2017 Apr;265(4):663-9.

40. Brandsma HT, Hansson BME, Aufenacker TJ, van Geldere D, van Lammeren FM, Mahabier C, et al.

Prophylactic mesh placement to prevent parastomal hernia, early results of a prospective multicentre randomized trial. Hernia. 2016 Aug;20(4):535-41.

41. Odensten $\mathrm{C}$, Strigård K, Rutegård J, Dahlberg M, Ståhle U, Gunnarsson U, et al. Use of Prophylactic Mesh When Creating a Colostomy Does Not Prevent Parastomal Hernia: A Randomized Controlled Trial-STOMAMESH. Annals of Surgery. 2019 Mar;269(3):427-31.

42. Janson AR, Jänes A, Israelsson LA. Laparoscopic stoma formation with a prophylactic prosthetic mesh. Hernia. 2010 Oct;14(5):495-8.

43. Price TJ, Beeke C, Ullah S, Padbury R, Maddern G, Roder D, et al. Does the primary site of colorectal cancer impact outcomes for patients with metastatic disease? Cancer. 2015 Mar 15;121(6):830-5.

44. Peralta R, Latifi R. Long-term outcomes of abdominal wall reconstruction. what are the real numbers? World J Surg. 2012 Mar;36(3):534-8.

\section{Tables}

Table 1: Baseline Demographics and Clinicopathological Characteristics between the experimental and control group

\begin{tabular}{|c|c|c|c|}
\hline Characteristics & Experimental $(n=24)$ & Control $(n=32)$ & P-value \\
\hline Age (year) n (\%) & $65.42(12.9)$ & $65.53(11.8)$ & 0.97 \\
\hline \multicolumn{4}{|l|}{ Gender } \\
\hline Female, n (\%) & $6(25)$ & $12(37)$ & \multirow[t]{2}{*}{0.48} \\
\hline Male, n (\%) & $18(75)$ & $20(63)$ & \\
\hline \multicolumn{4}{|c|}{ AJCC Staging, $n(\%)$} \\
\hline Stage I & $6(25)$ & $8(25)$ & \multirow[t]{4}{*}{0.7304} \\
\hline Stage II & $9(37.5)$ & $8(25)$ & \\
\hline Stage III & $9(37.5)$ & $15(46.87)$ & \\
\hline Stage IV & & $1(3.13)$ & \\
\hline
\end{tabular}

Page $13 / 18$ 
Figures

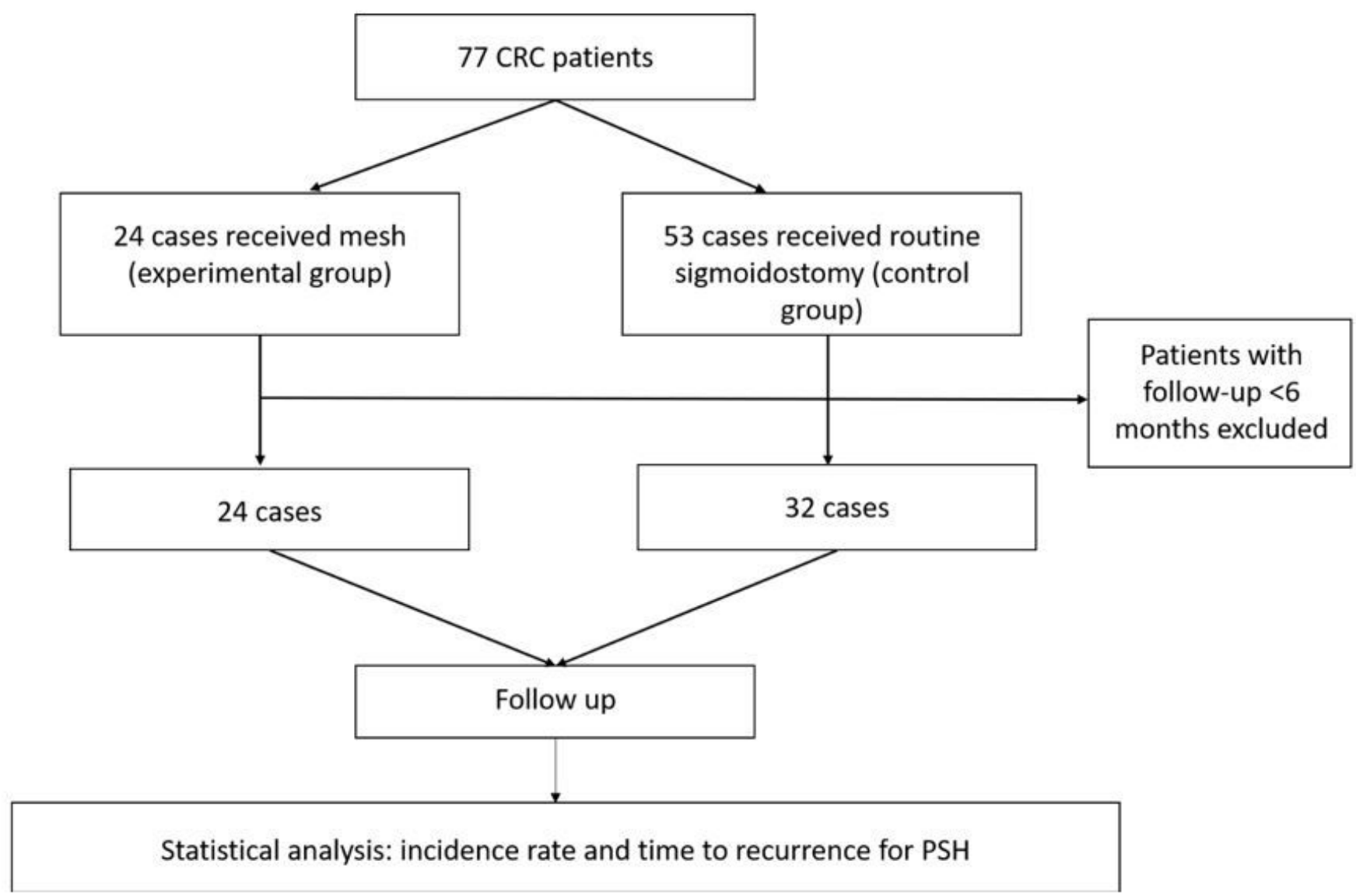

Figure 1

Patient disposition at baseline 


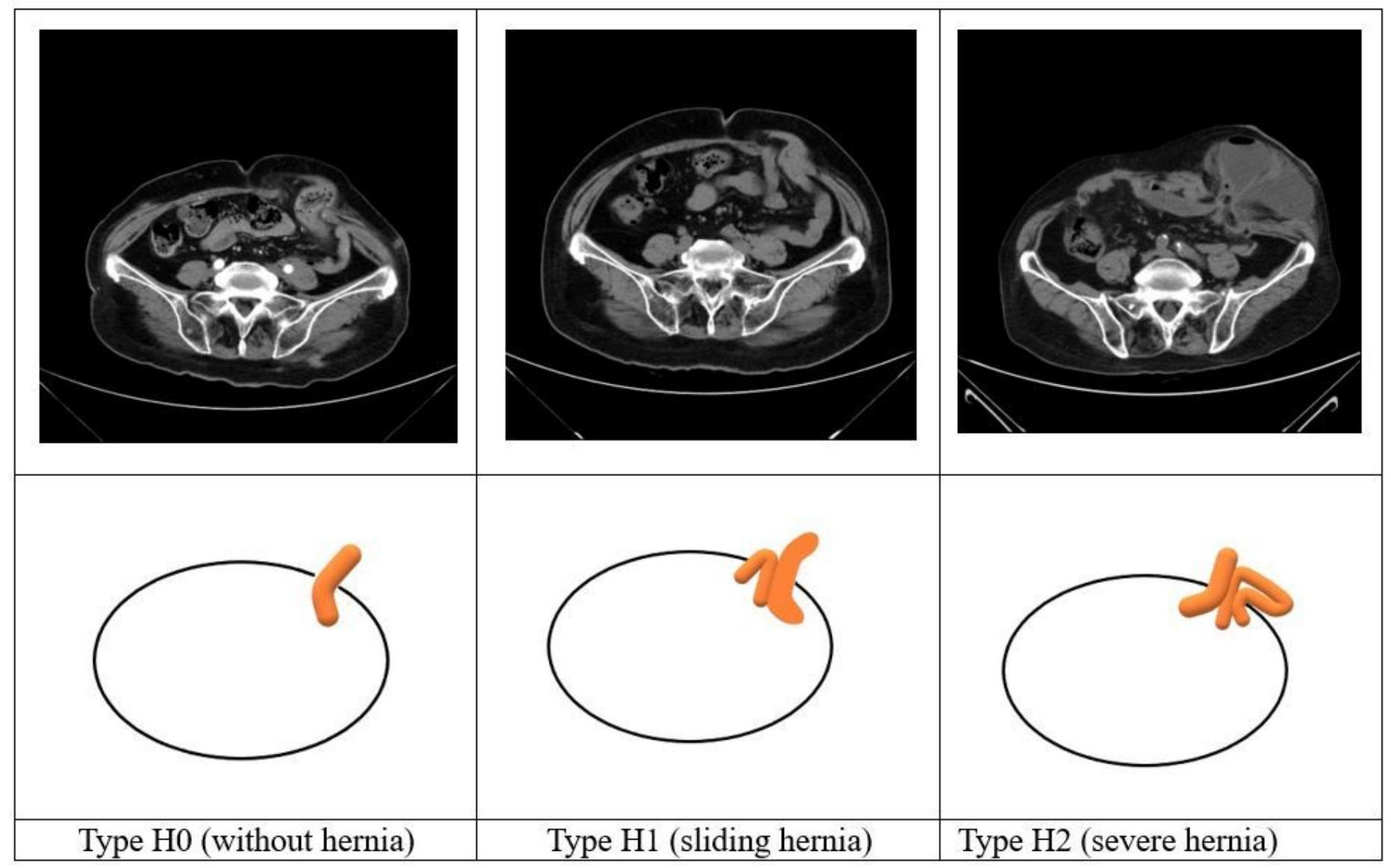

Figure 2

Classification of different types of parastomal hernia 


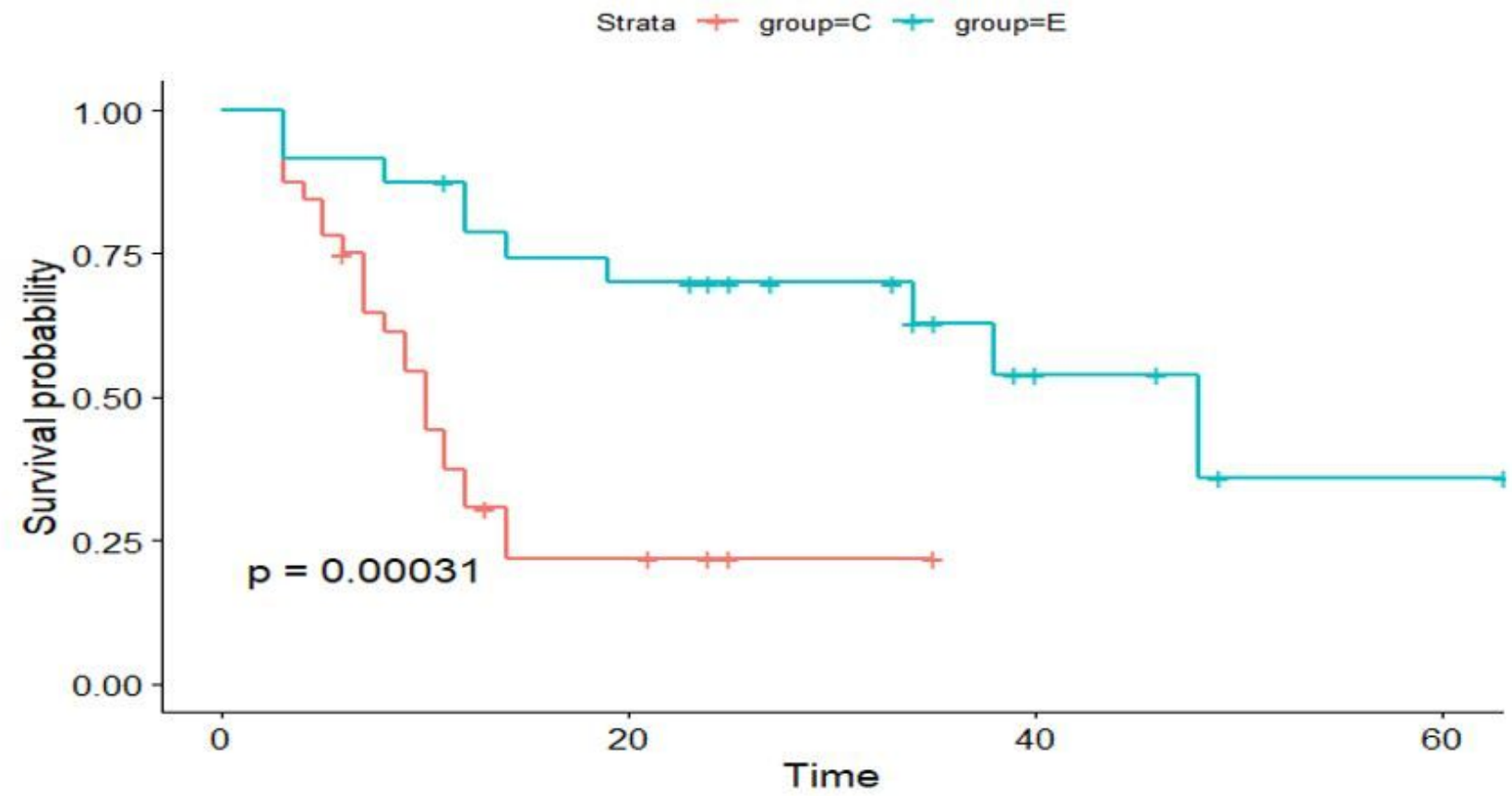

C: control; E: experimental

Figure 3

Postoperative time to $\mathrm{PSH}$ recurrence 


\section{cumulative incidence by Group}

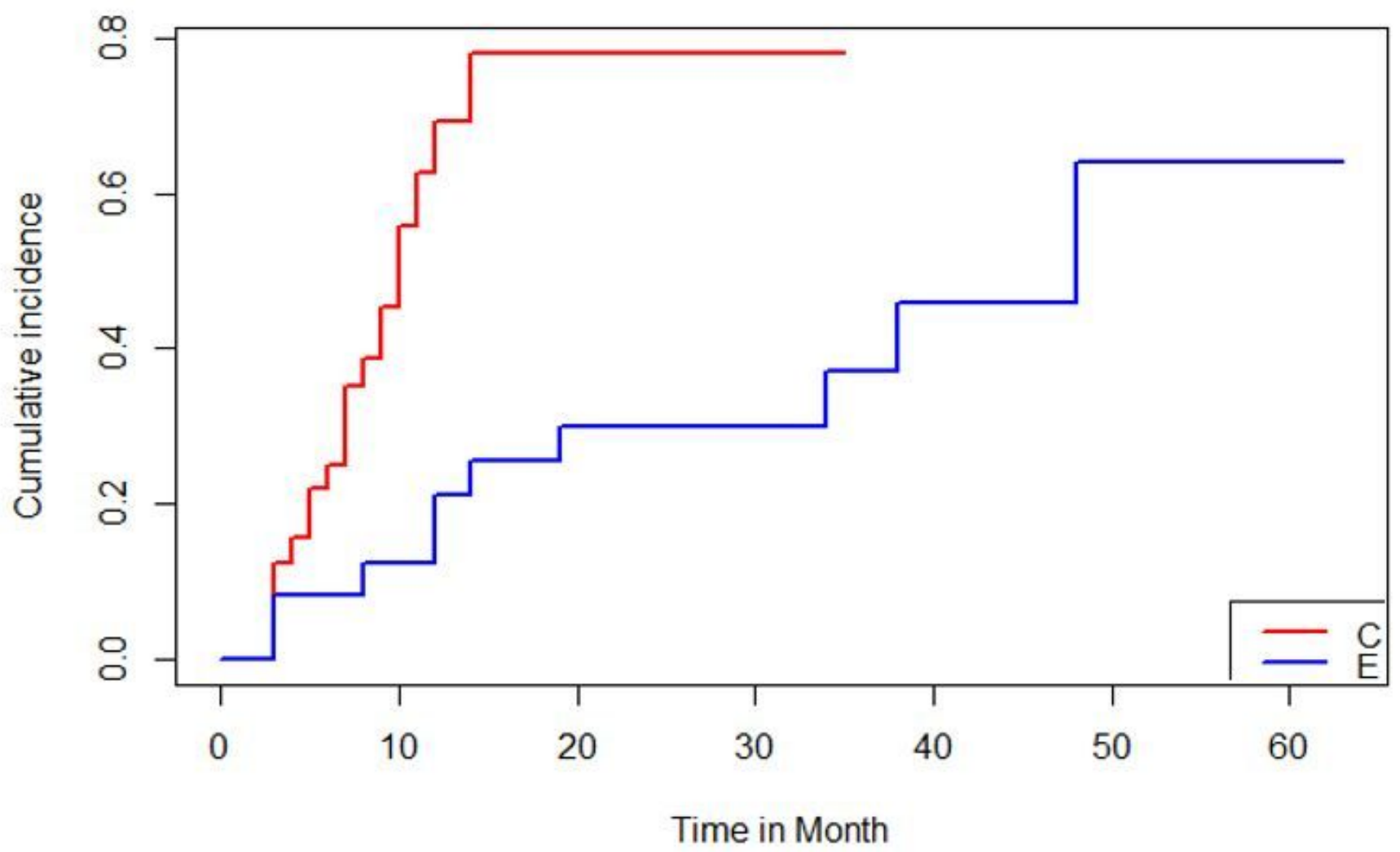

C: control; E: experimental

Figure 4

Cumulative incidence of PSH 


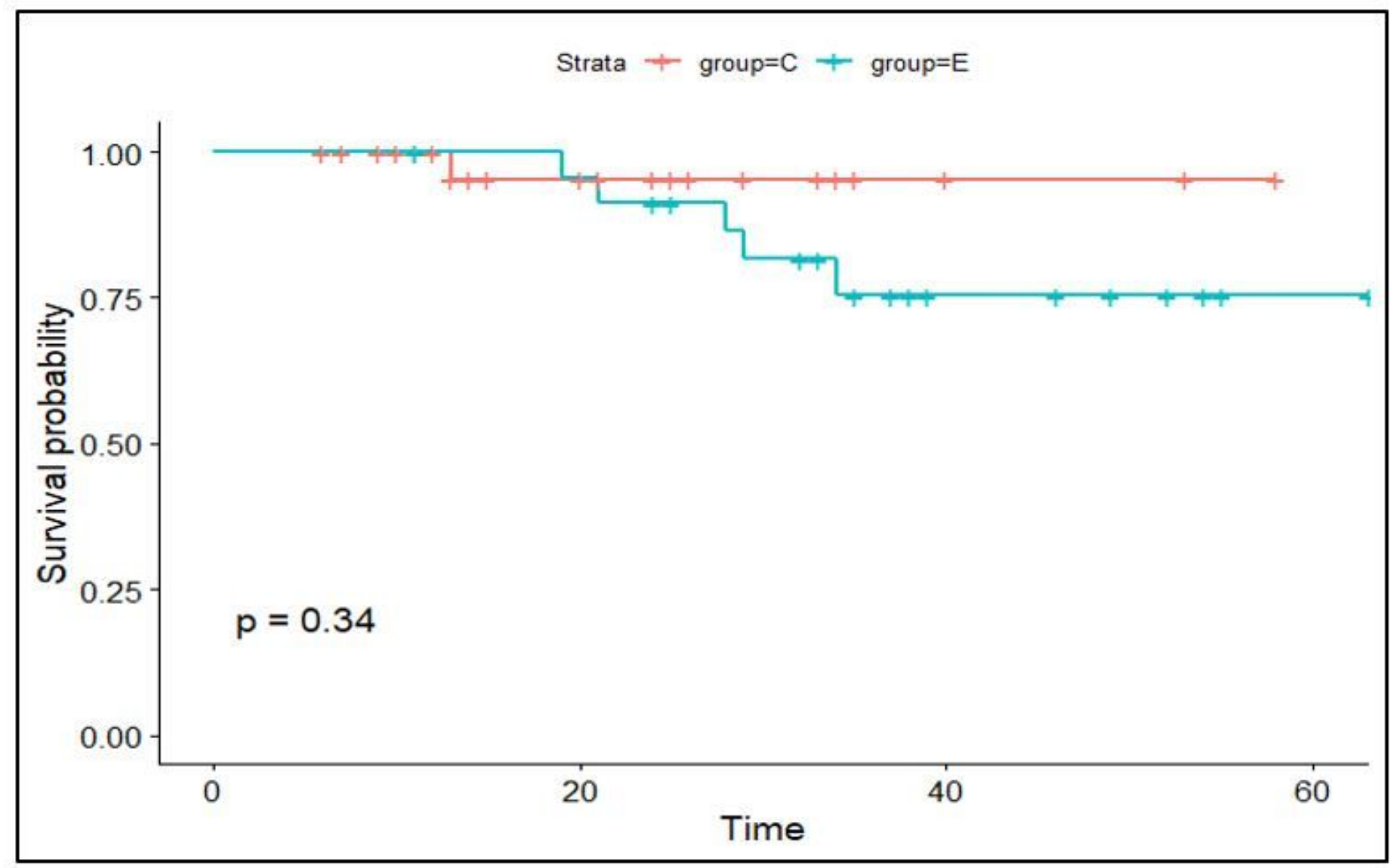

Figure 5

$\mathrm{KM}$ curves for survival time 